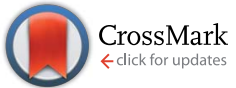

Cite this: RSC Adv., 2017, 7, 5030

\title{
Improved viscoelastic, thermal, and mechanical properties of in situ microfibrillar polypropylene/ polyamide 6,6 composites via direct extrusion using a triple-screw extruder
}

\author{
Ying Huang, ${ }^{a}$ Yadong He, ${ }^{\text {ab }}$ Weidan Ding, ${ }^{\mathrm{c}}$ Kunxiao Yang, ${ }^{\mathrm{a}}$ Dongquan $\mathrm{Yu}^{\mathrm{a}}$ \\ and Chunling Xin*ab
}

In this work, a triangle arrayed triple-screw extruder (TTSE) was used to prepare in situ polypropylene/ polyamide 6,6 (PP/PA66) microfibrillar composites (MFCs) by direct extrusion at a processing temperature between the melting points of the two phases. The dispersed phase, PA66 particles, deformed, merged and was stretched into fibrils under the alternating shearing and extensional flow field in the TTSE. The fibrillar morphology was controlled through adjusting the PA66 content and processing parameters (temperature and screw speed). Dynamic oscillatory shear rheological properties and extensional viscosity of PP/PA66 microfibrils with different aspect ratio were studied. The obtained results showed that the storage modulus and complex viscosity of PP/PA66 MFCs were improved with increasing fibrillar aspect ratio. Meanwhile, the loss $\operatorname{tangent} \tan \delta$ value decreased and the radius of the Cole-Cole plot circle increased with an increase in fibrillar aspect ratio. The PP/PA66 MFCs exhibited a pseudo-solid or gel-like behavior. The gel point concentration, which was determined by the WinterChambon criterion, decreased with the increasing fibrillar aspect ratio due to the increased interfacial area and enhanced entangled network structure. Additionally, high-aspect-ratio PA66 fibrils have dramatically improved PP's melting temperature, crystallization kinetics, and mechanical properties.

Received 13th November 2016 Accepted 31st December 2016

DOI: $10.1039 / c 6 r a 26734 c$

www.rsc.org/advances fibrillation of polymer blend, which converts the dispersed particles into micro- or nano-fibrillar structure, has attracted much attention..$^{3-8}$ Owing to the flexibility and high aspect ratio, the fibrils can deform adequately in response to interfibrillar interactions during flow. ${ }^{9}$ When the fibrils concentration exceeds a certain value, the deformed fibrils can form a physical network structure which renders the blends excellent properties. The presence of such an entangled fibrillar network has greatly enhanced MFCs' crystallization kinetics ${ }^{10-12}$ and mechanical properties. ${ }^{8,13,14}$ For instance, Zhao et al. ${ }^{10}$ found that the presence of the PET fibrillar network had significantly improved the crystal nucleation ability of iPP, especially for the long PET fibers. The study conducted by Yang et al. ${ }^{13}$ showed that the mechanical performance was significantly improved by the in situ PA6 microfibrils and special crystalline structures.

Many studies have also been carried out to explore the influence of in situ microfibrils on the polymers' rheological properties such as shear and elongational viscoelastic behavior. ${ }^{15-19}$ Currently, it has been acknowledged that the presence of a physical entanglement network defined by topological (entangled) interactions can significantly enhance the composite's melt elasticity, and thus, affect the material properties and its processability. The enhanced melt elasticity is very important for some processing such as blow molding and
Technology, Beijing 100029, China. E-mail: xincl@mail.buct.edu.cn

${ }^{b}$ Engineering Research Center for Polymer Processing Equipment, Ministry of Education, Beijing 100029, China

${ }^{c}$ Dart Container, 500 Hogsback Road, Mason, MI 48854, USA 
foaming, where biaxial strength is needed. ${ }^{11}$ Dong et al. ${ }^{6}$ observed that the poly(trimethylene terephthalate) (PTT) microfibrils had a greater influence on the rheological properties of polyolefin elastomer (POE) than PTT particles because the PTT microfibrils could form a physical network within the POE matrix. Zhao et al. ${ }^{\mathbf{1 0}}$ and Wang et al. ${ }^{8}$ prepared in situ MFCs with different methods and measured the effects of dispersed phase concentration on the complex viscosity. Both studies illustrated that the microfibrils are conducive to increase complex viscosity of polymer matrix. Jayanarayanan et al. ${ }^{\mathbf{2 0}}$ found that the storage modulus, loss modulus and complex viscosity of $\mathrm{PP} / \mathrm{PET}$ MFCs were enhanced as draw ratio increased up to an optimized level. The authors attributed the increase in the viscosity to the formation of a physical PET fibril network which hinders the relaxation of the PP phase, as indicated from the frequency-independent $\tan \delta$ curve.

Generally, the method of Winter-Chambon analysis ${ }^{\mathbf{2 1 , 2 2}}$ is used to identify the gel point, ${ }^{9}$ that is, the critical fibril content at which a rheologically percolated network forms. The formation of such a network is a typical characteristic of the composites with deformable and long (that is, high aspect ratio) fibers. For example, Kakroodi et al. ${ }^{\mathbf{1 1}}$ and Rizvi et al. ${ }^{\mathbf{1 6}}$ applied this method and found out that $3.0 \mathrm{wt} \%$ PA and $4.5 \mathrm{wt} \%$ PP were needed for the PLA and PE systems, respectively. The strain hardening behavior of polymer blends with fibrillar morphology has been reported, and the dramatic improvements in strain hardening response of polyethylene (PE) was observed through the in situ fibrillation of $1 \mathrm{wt} \% \mathrm{PP} .^{23}$

Different MFC systems have been studied to demonstrate the significant effect of fibrillar structure on the properties of the polymer matrix, including PLA/PA, ${ }^{11} \mathrm{PP} / \mathrm{PET},{ }^{12,20} \mathrm{PE} / \mathrm{PP}^{16}$ and POE/PTT. ${ }^{6}$ In these studies, fibril concentration was taken as the governing factor affecting the formation of a fibrillar network. However, it is well known the network structure is not only dependent on the fibril concentration, but also on the fibrillar morphology such as its dispersion, diameter, aspect ratio, and deformability. ${ }^{24}$ So far, no study has been found on the influence of specific fibrillar aspect ratio on the formation of network structure. Therefore, it became the primary objective of this study.

Currently, most of the microfibrillar composites are prepared by melt extrusion, continuous hot stretching, and quenching process. ${ }^{5,11,25}$ That is, three steps should be adopted: (1) melt extrusion: melt blending of the dispersed phase and matrix phase at a temperature above the melting point of the dispersed phase; (2) continuous hot stretching: drawing the extrudate to fibrillate the dispersed component at a temperature between the melting temperature of dispersed phase and matrix phase; and (3) isotropization of the oriented blend. Although this processing method has been widely used for preparation of MFCs, it is undeniable that it is complicated and involves multiple stages. Rizvi et al. ${ }^{12,16}$ attempted to adopt another method to prepare microfibrillar composites. In their work, the polymers were first melt compounded at a high temperature in a co-rotating twin-screw extruder with a built-in back-flow channel which enables material recirculation for several minutes, and followed by a low-temperature mixing for extra minutes before drawing. Despite that the above process was successful in producing the high aspect ratio fibril, the polymers were retained in the extruder for quite a long time and the productivity was low. Thus, it is not suitable for thermalsensitive polymers and is inefficient for industrial production. Jurczuk et al. ${ }^{26}$ demonstrated that compounding molten polymer matrix with solid state polytetrafluoroethylene (PTFE) powder in co-rotating twin-screw was effective to in situ fabricate the fibrils. As far as we know, almost none exploration of utilizing the deformation of other thermoplastic polymers into nano- or micro-fibers such as PET/PBT/PA6/PA66 for microfibrillar composites by this mechanism had been investigated.

Triangle arrayed triple-screw extruder (TTSE) is a novel type of polymer processing equipment with special mixing characteristics and high productivity. It consists of three meshing zones and a central region and generates greater shearing and extensional flow field for polymer compounding. ${ }^{27-29}$ In this work, we demonstrated that a single extrusion process with TTSE was effective to prepare polyamide 6,6 (PA66) microfirbils in PP polymer matrix without post-extrusion drawing. By varying the processing conditions, in situ PP/PA66 MFCs with well-dispersed high aspect ratio PA66 fibrils were prepared. After that, the dynamic rheological behaviors and uniaxial extensional rheological behaviors of PP containing various aspect ratio fibrils were investigated. The gel point of PP/PA66 MFCs with different aspect ratio PA66 fibrils were thoroughly analyzed according to the Winter-Chambon criterion. Furthermore, the effects of fibrillar morphology and fibril content on the thermal and mechanical properties were studied.

\section{Experimental}

\subsection{Materials}

iPP T03-H (Sinopec Yangzi, China) is the commercial product of Sinopec Yangzi Petrochemical. It has a density of $0.91 \mathrm{~g} \mathrm{~cm}^{-3}$ with a melt flow rate (MFR) of $3.6 \mathrm{~g} / 10 \mathrm{~min}$ at $230{ }^{\circ} \mathrm{C} / 2.16 \mathrm{~kg}$. Polyamide 6,6 (PA66), a typical engineering thermoplastic material, was used as the minor phase in this study. It is supplied by Shenma Group Co., Ltd, China with a density of $1.15 \mathrm{~g} \mathrm{~cm}^{-3}$ and an intrinsic viscosity of $2.7 \mathrm{dl} \mathrm{g}^{-1}$. The melting temperatures $\left(T_{\mathrm{m}}\right)$ of iPP and PA66 were found to be $162^{\circ} \mathrm{C}$ and $263^{\circ} \mathrm{C}$ respectively, using Differential Scanning Calorimetry (DSC) under a nitrogen atmosphere with a heating rate of $10{ }^{\circ} \mathrm{C} \mathrm{min}^{-1}$.

\subsection{Sample preparation}

The in situ PP/PA66 MFCs were fabricated through a TTSE equipped with three inverted triangle arrayed screws (the screw diameter of extruder was $35.2 \mathrm{~mm}$, with aspect ratio of 28). The scheme of the experimental setup for the microfibrillar composites preparation is shown in Fig. 1. There were seven temperature zones from hopper to the extruder die. The temperature increased gradually from $170{ }^{\circ} \mathrm{C}$ to working temperature along the extruder and kept constant at the last four temperature zones. Subsequently, the extrudate was immediately quenched in cold water $\left(20{ }^{\circ} \mathrm{C}\right)$ to prevent the retraction of fibrils, and the granulator speed kept consistent with the extrusion speed. Different PA66 content and processing 
$\operatorname{Tm}(\mathrm{PP})<\mathrm{T}<\operatorname{Tm}(\mathrm{PA66})$

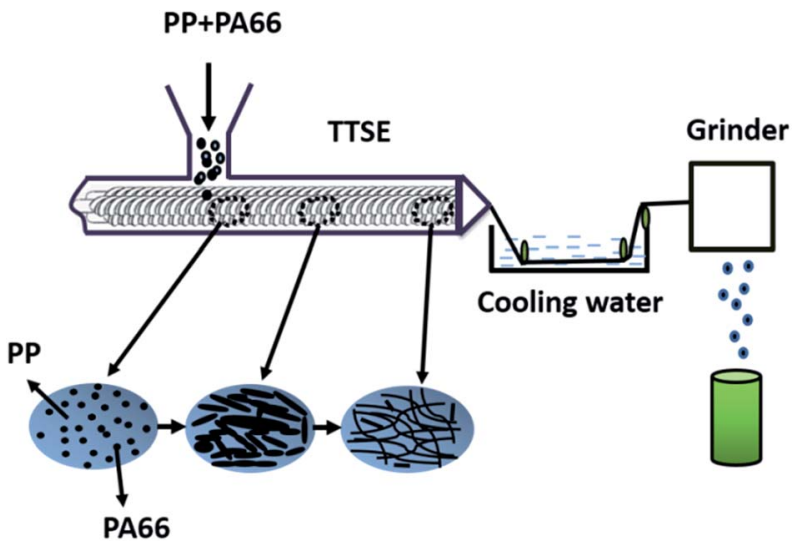

Fig. 1 The schematic of the experimental set-up for the in situ microfibrillar composites preparation.

parameters (temperature and screw speed) owning three levels were introduced to prepare PP/PA66 MFCs with different PA66 phase morphology. LC, MC, and HC denote low, medium, and high PA66 content $(10,20$ and $30 \mathrm{wt} \%)$, respectively. LT, MT, and HT denote low, medium, and high processing temperature (230, 245 and $\left.260{ }^{\circ} \mathrm{C}\right)$, respectively. LS, MS, and HS denote low, medium and high screw speed (100, 150 and $200 \mathrm{rpm}$ ), respectively. Without special illustrating, the medium level processing parameters were adopted. Additionally, the normal blends (NB) were obtained when the PA66 content, processing temperature and screw speed were $20 \mathrm{wt} \%, 275^{\circ} \mathrm{C}$, and $200 \mathrm{rpm}$ respectively.

An injection molding machine SA900/260 (Ningbo Haitian, China) was used to prepare specimens for mechanical test. The temperature profiles from hopper to nozzle of the injection molding machine were controlled at 170, 190, 210, 210 and $205{ }^{\circ} \mathrm{C}$. The low processing temperature was used to preserve the original fibrillar morphology of PA66 in the composites. The internal mixer was employed to dilute the masterbatch samples into different fibril contents with the temperature, screw speed, and mixing time of $180{ }^{\circ} \mathrm{C}, 40 \mathrm{rpm}$, and $4 \mathrm{~min}$, respectively.

\subsection{Morphological characterization}

The morphology of the blends was observed using a scanning electron microscope (SEM, HITACHI S 4700). Prior to the observation, the samples were subjected to solvent vapor etching process using xylene for $12 \mathrm{~h}$ at $140{ }^{\circ} \mathrm{C}$ to extract PP phase. The etched samples were coated with a thin gold layer prior to the SEM analysis. Image-Pro Plus 6.0 were used to characterize the fibrillar morphology (diameter and length) from SEM photographs. Approximately 200 fibrils for each sample were characterized for statistical analysis of the fibrillar diameter and aspect ratio.

\subsection{Rheological measurements}

The dynamic linear viscoelastic behaviors of neat PP and PP/ PA66 blend with spherical and fibrillar PA66 domains were studied using a parallel-plate rotational rheometer (HAAKE MARS III). The diameter of the plate was $25 \mathrm{~mm}$ and the gap between plates was $1 \mathrm{~mm}$. In order to maintain the PA66 fibrils in the composites, the test temperature for all samples were kept at $190{ }^{\circ} \mathrm{C}$. The frequency varied from 0.05 to $500 \mathrm{rad} \mathrm{s}^{-1}$, using $0.1 \%$ strain value determined by a strain sweep to keep within the linear viscoelastic region.

Measurements of uniaxial extensional viscosity were made using a Sentmanat Extensional Rheometer (SER) attached to the HAAKE MARS III. Samples were tested at strain rates $0.05 \mathrm{~s}^{-1}$ and at a sample environment temperature of $170{ }^{\circ} \mathrm{C}$. We empirically found that this temperature provided us with the most reproducible and consistent results.

\subsection{Differential scanning calorimetry (DSC) measurements}

The melting temperature and nonisothermal crystallization behavior of PP containing both spherical and microfibrillar domains were measured using a differential scanning calorimeter (DSC-60, Shimadzu Corporation) in nitrogen atmosphere. The samples were first heated up from 40 to $200{ }^{\circ} \mathrm{C}$ and then hold for $5 \mathrm{~min}$ to eliminate the influence of thermal history, following cooled down to $50{ }^{\circ} \mathrm{C}$ at a predetermined constant rate of $10{ }^{\circ} \mathrm{C}$ $\min ^{-1}$ for nonisothermal crystallization. The melting point of $\mathrm{PP}$ phase was determined during second heating of the samples to $200{ }^{\circ} \mathrm{C}$ at a heating rate of $10{ }^{\circ} \mathrm{C} \mathrm{min}^{-1}$.

\subsection{Mechanical measurements}

Uniaxial tensile properties of polymer blends were measured at room temperature using an Instron 4302 universal tensile test. All the tests were conducted at a constant crosshead speed of 20 $\mathrm{mm} \min ^{-1}$ according to ASTM D638 standard. Izod impact strength was performed using an impact tester (ZBC1400-2) with specimen dimension of $80 \times 10 \times 4 \mathrm{~mm}^{3}$ according to ASTM D256-2003. The presented value was the average of five individual measurements.

\section{Results and discussion}

\subsection{Morphological characterization of fibrils}

PP/PA66 MFCs were prepared at different processing conditions and the effect of PA66 content, processing temperature, and screw speed on the formation of PA66 fibrils were studied. The sample codes representing the different conditions are listed in Table 1.

The influence of PA66 content $(10,20$, and $30 \mathrm{wt} \%)$ on fibrillar morphology at processing temperature of $245{ }^{\circ} \mathrm{C}$ and screw speed of $150 \mathrm{rpm}$ is presented in Fig. 3. Average diameter and average aspect ratio of PA66 fibrils at different processing conditions are summarized in Table 1 and Fig. 2. Compared to low temperature extrusion (Fig. 3a-c), no fibril was formed in PP/PA66 blends obtained by normal extrusion (Fig. 3d). As the PA66 content increased from $10 \mathrm{wt} \%$ to $20 \mathrm{wt} \%$, the average diameter of fibrils decreased from $4.51 \mu \mathrm{m}$ to $2.17 \mu \mathrm{m}$ and then rose to $4.02 \mu \mathrm{m}$ when the PA66 content further increased to 30 $\mathrm{wt} \%$. However, the aspect ratio rose significantly with an increase in the PA66 content, which was mainly attributed to 
Table 1 The diameter and aspect ratio of NB and PA66 microfibrils obtained at different conditions

\begin{tabular}{lllll}
\hline Sample code & Processing parameter $^{a}$ & Diameter range $(\mu \mathrm{m})$ & Average diameter $(\mu \mathrm{m})$ & Average aspect ratio \\
\hline MC-LT & $20 / 230 / 150$ & $1.22-8.57$ & $2.28 \pm 0.39$ & $73 \pm 10$ \\
MC-MT & $20 / 245 / 150$ & $0.84-5.39$ & $2.17 \pm 0.25$ & $128 \pm 8$ \\
MC-HT & $20 / 260 / 150$ & $1.15-7.32$ & $2.76 \pm 0.72$ & $42 \pm 23$ \\
LC-MT & $10 / 245 / 150$ & $1.30-8.57$ & $4.51 \pm 0.47$ & $13 \pm 5$ \\
HC-MT & $30 / 245 / 150$ & $1.68-8.84$ & $4.02 \pm 0.31$ & $210 \pm 11$ \\
HC-LS & $30 / 245 / 100$ & $1.77-8.68$ & $4.83 \pm 0.65$ & $178 \pm 13$ \\
HC-HS & $30 / 245 / 200$ & $1.93-7.95$ & $3.70 \pm 0.27$ & $225 \pm 20$ \\
NB & $20 / 275 / 150$ & $0.84-6.13$ & $3.08 \pm 0.31$ & 1
\end{tabular}

${ }^{a}$ Note: the processing parameters represent PA66 mass content/processing temperature/screw speed.

the fact that higher chance for the dispersed phase to coalescence occurred in the extruder flow field with the increasing PA66 content.

Fig. 4a-c demonstrates the PA66 morphology after extraction of PP prepared under low, medium and high temperature (230, 245 and $260{ }^{\circ} \mathrm{C}$ ). The observation of diameter and aspect ratio of the fibril revealed that the medium temperature $\left(245{ }^{\circ} \mathrm{C}\right)$ was favorable for the formation of high-aspect-ratio fibrils (Fig. 4b). Softening and maintaining deformation of particles were tremendously affected by temperature. At the low processing temperature $\left(230^{\circ} \mathrm{C}\right)$, PA granules were not softened enough to be stretched into long fibrils (Fig. 4a). On the contrary, at $260^{\circ} \mathrm{C}$, which is close to the melting temperature of PA66, the PA molecular chains tend to relax and recoil back after stretching in the extruder, resulting in greater and non-uniform diameters (Fig. 4c) compared to the low-temperature cases.

Additionally, the effects of the screw speed on the morphology of fibrils with the PA66 content $30 \mathrm{wt} \%$ and processing temperature of $245{ }^{\circ} \mathrm{C}$ were studied, and the results are shown in Fig. 4d-f. It can be seen that, at medium processing temperature, all three screw speeds could generate high-aspectratio fibrils and higher screw speed producing thinner and higher-aspect-ratio fibrils (Fig. 2). It is understandable that higher shear generated from higher screw speed helped the PA66 domains deform into much uniform and smaller fibrils.

Such a preparation method of in situ microfibrillar composites has been proved to be exercisable by the evidence of fibrillar morphology with wonderful dimension parameters without drawing. The formation mechanism of fibrils in TTSE can be interpreted from two aspects. On the one hand, the spherical particles convert into ellipsoid primarily under strong shearing and drawing field of the extruder and following ellipsoid bond with other similar neighbors, eventually forming large aspect ratio and uniform fibrils suffered from the shearing and extensional flow field. ${ }^{30}$ On the other hand, another part of the dispersed phase form sheets or blocks from starting, breaking into a network and fibrous structures through interfacial force because of the instability of sheets or blocks. ${ }^{31}$

\subsection{Rheological properties}

In order to investigate the effect of fibrils' aspect ratio on the rheological properties of PP/PA66 MFCs, the masterbatch samples with aspect ratio of 42 (AR42), 73 (AR73), 128 (AR128), 178 (AR178), 210 (AR210) and 1 (NB) (Table 1) were diluted with neat PP to a final PA content of $5 \mathrm{wt} \%$.

Fig. 5 shows the shear responses of molten PP and the PP/ PA66 compounds with spherical and PA66 fibrils at $190{ }^{\circ} \mathrm{C}$. Fig. 5a and b illustrate storage modulus $\left(G^{\prime}\right)$ and complex viscosity $\left(\eta^{*}\right)$ as a function of frequency for MFCs with different fibrillar aspect ratio ranging from 1 to 210 . The increasing of the aspect ratio leads to a gradual increase in both $G^{\prime}$ and $\eta^{*}$ but the increasement in $G^{\prime}$ is more pronounced than that in $\eta^{*}$. Furthermore, the storage modulus $\left(G^{\prime}\right)$ had strong dependence on the fibrillar aspect ratio at low frequency. Interestingly, $G^{\prime}$ becomes increasingly independent of frequency as the fibrillar aspect ratio increased, which is consistent with the phenomenon that $G^{\prime}$ showed increasingly independent of $\omega$ as the fibril content increased in previous studies. ${ }^{16,32}$ Both indicated that the viscoelastic response was changing from 'liquid-like' to 'gellike'.$^{33,34}$ The plot of $\tan \delta-\omega$ is shown in Fig. 5c. Tan $\delta$ decreased with fibrillar aspect ratio increasing. Specially, for the blend with fibrillar aspect ratio of $210, \tan \delta$ nearly exhibited frequency independence. Such an increase in the melt's elastic behavior suggests the formation of a physically entangled network, which would have been created by the topological interactions of the PA66 fibrils. ${ }^{35}$
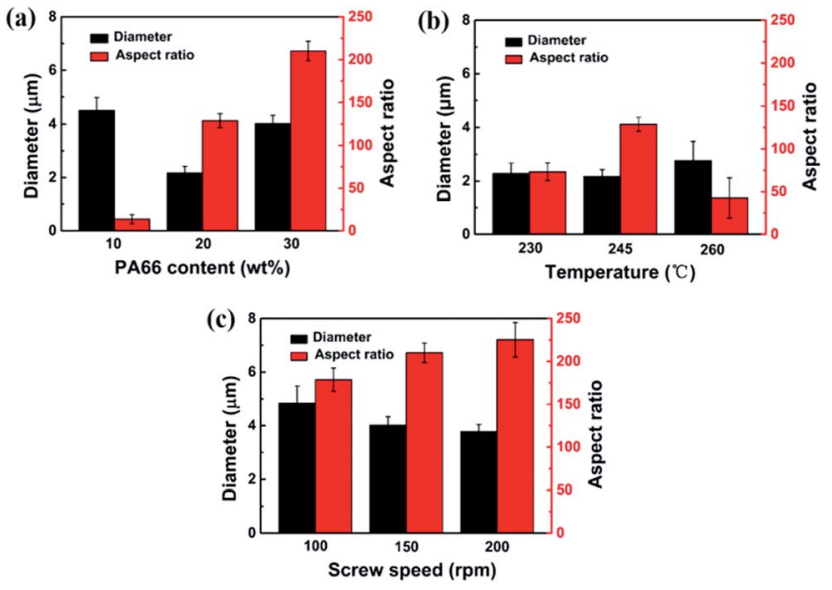

Fig. 2 The effect of (a) PA66 content, (b) processing temperature and (c) screw speed on the average diameters and aspect ratios of PA66 fibrils. 

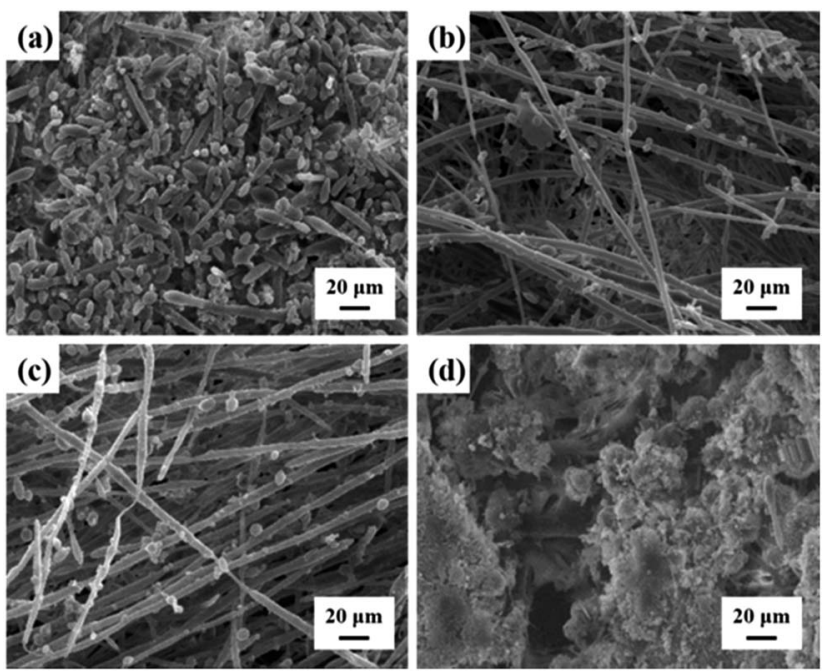

Fig. 3 Morphology of in situ PA66 fibril with different PA66 content after extraction of PP: (a) LC-MT; (b) MC-MT; (c) HC-MT; (d) NB.

The Cole-Cole plot (i.e., dynamic viscosity $\eta^{\prime \prime}$ versus $\eta^{\prime}$ ) was often used to characterize the relaxation mechanism of longchain branched molecular chain under small amplitude oscillatory shear. ${ }^{36}$ A larger radius of Cole-Cole circle suggested a longer relaxation time. For long-chain branched polymers, the relaxation was primarily governed by the degree of entanglement of molecular chains; and a higher degree of entanglement resulted in a longer relaxation time. Similarly, in PP/PA66 MFCs, a longer relaxation time indicated a higher degree entanglement of PA66 fibrils. As shown in Fig. 5d, the radius of ColeCole circle increases as the fibrillar aspect ratio increases. Therefore, we concluded that higher aspect ratio had formed a higher degree of entangled network structure at the same PA content.

Fig. 5 confirms that the fibrillar aspect ratio significantly influenced the formation of physical entanglement network structure. The structural implication of the enhanced elasticity observed with the increase in aspect ratio was that the solidstate PA66 fibrils tended to restrict the long range motion of the matrix polymer chains and prevents them from complete
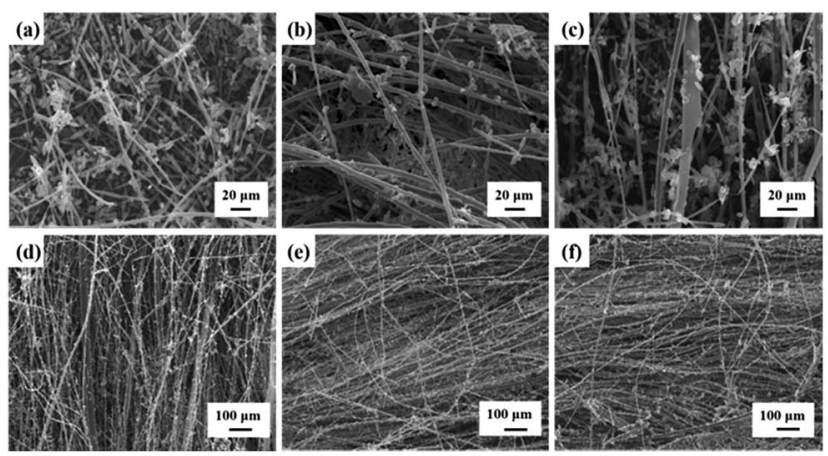

Fig. 4 Morphology of PP/PA66 blends at different processing temperature (a) MC-LT; (b) MC-MT; (c) MC-HT and screw speeds (d) $\mathrm{HC}-\mathrm{LS}$; (e) HC-MT; (f) HC-HS after extraction of PP. relaxation when subjected to external force. ${ }^{\mathbf{1 2}}$ The WinterChambon criterion had been widely used to accurately determine the gel point of physical and chemical gels from rheological data. A multi-frequency plot of $\tan \delta$ versus the fibril content revealed an intersection that precisely marked the gel point, as shown in Fig. 6. We observed that, for a specific fibrillar aspect ratio, the plots of $\tan \delta$ at different frequencies gradually decreased with an increase in fibril content and intersected at one fibril content. And this fibril content was considered as the gel point beyond which a rheological percolated fibrillar network was formed in the system regardless of the applied frequency. This was attributed to the magnitude change of $G^{\prime}(\omega)$ and $G^{\prime \prime}(\omega)$ before and after gel point at different $\omega$. Thus, the Winter-Chambon theory was also applicable to find the gel points of the PP/PA66 microfibrillar composites.

As shown in Fig. $6 \mathrm{a}_{1}$, the curves of $\tan \delta$ intersect at a fibril content of $7.0 \mathrm{wt} \%$, thus, the gel point concentration can be accurately identified to be $7.0 \mathrm{wt} \%$ for the PP/PA66 MFCs with fibrillar aspect ratio of 42. Similarly, the gel point of AR73, AR128, AR178 and AR210 were determined as 6.7, 5.9, 4.5 and $3.8 \mathrm{wt} \%$ (Fig. $6 \mathrm{~b}_{1}-6 \mathrm{e}_{1}$ ), respectively. This phenomenon could be concluded that the fibrillar aspect ratio indeed significantly affected the formation of the gel point. Moreover, the higher aspect ratio was, the lower fibril content was required to form the physical entanglement network (Fig. 7a). The fibril content was reduced from 7.0 to $3.8 \mathrm{wt} \%$ when the fibril aspect ratio increased from 42 to 210 . This could be due to two aspects: on the one hand, fibrils with higher aspect ratio have greater surface area, thus increasing the interfacial area between PP molecular chains and the PA fibrils. On the other hand, the high-aspect ratio PA fibrils tended to form a higher degree of entanglement than low-aspect-ratio ones.

The zero shearing viscosity $\eta_{0}$ and relaxation time $\lambda$ of PP containing PA66 fibril with different fibril content and aspect ratio were obtained through fitting the plot of $\lg \eta^{*}-\lg \omega$ based on Carreau model ${ }^{37}$ and the effect of aspect ratio on $\eta_{0}$ and $\lambda$ is shown in Fig. $7 \mathrm{~b}$ and c. According to Doi-Edwards model, ${ }^{38}$ the
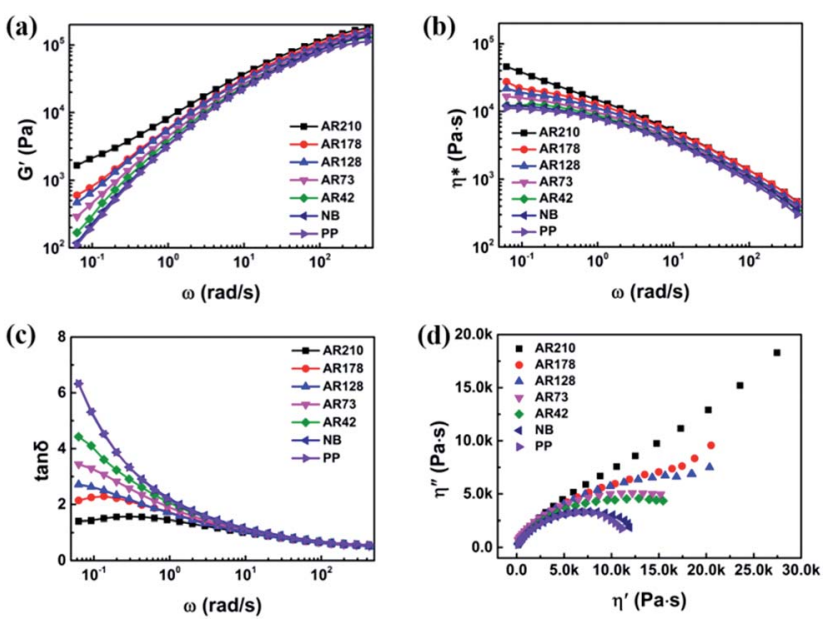

Fig. 5 Effect of fibrillar aspect ratio on (a) the storage modulus $\left(G^{\prime}\right)$, (b) complex viscosity $\left(\eta^{*}\right)$, (c) loss tangent ( $\tan \delta$ ) and (d) dynamic viscosity $\left(\eta^{\prime \prime}\right)$ of the composites. 

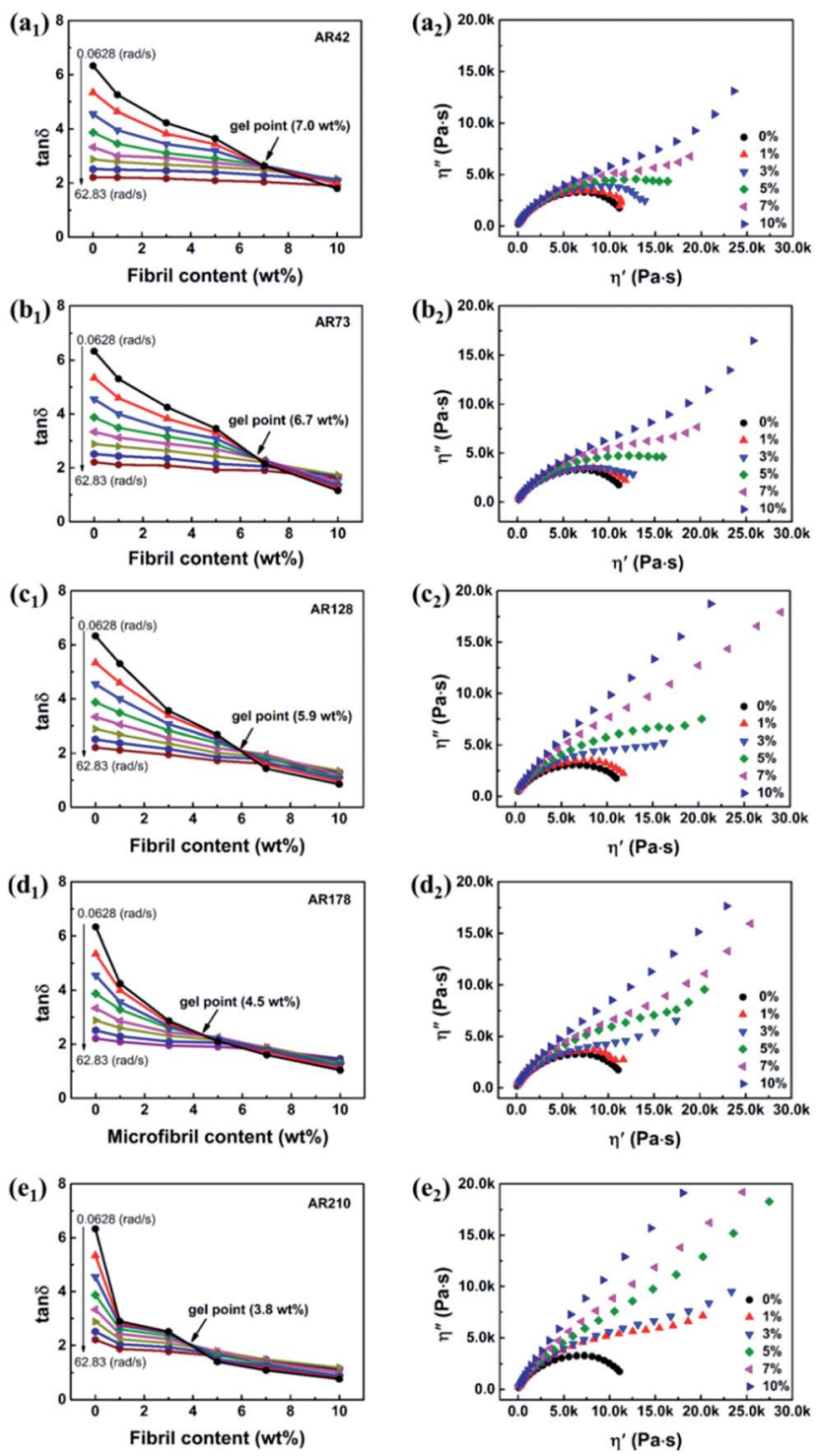

Fig. 6 Rheological behaviour of PP/PA66 microfibrillar blends at $190{ }^{\circ} \mathrm{C}$ for five samples with different PA66 aspect ratio: $\left(a_{1}\right) \tan \delta$ as a function of fibril content and $\left(a_{2}\right)$ dynamic viscosity $\left(\eta^{\prime \prime}\right)$ of AR42; $\left(b_{1}\right)$ $\tan \delta$ as a function of fibril content and $\left(\mathrm{b}_{2}\right)$ dynamic viscosity $\left(\eta^{\prime \prime}\right)$ of AR73; $\left(\mathrm{c}_{1}\right) \tan \delta$ as a function of fibril content and $\left(\mathrm{c}_{2}\right)$ dynamic viscosity $\left(\eta^{\prime \prime}\right)$ of AR128; $\left(\mathrm{d}_{1}\right) \tan \delta$ as a function of fibril content and $\left(\mathrm{d}_{2}\right)$ dynamic viscosity $\left(\eta^{\prime \prime}\right)$ of AR178; $\left(\mathrm{e}_{1}\right) \tan \delta$ as a function of fibril content and $\left(\mathrm{e}_{2}\right)$ dynamic viscosity $\left(\eta^{\prime \prime}\right)$ of AR210.

relaxation time can be used to characterize the material's elastic behaviour and it exhibited an exponential increase with the growth of branching chain length and entanglement. It was noticed that both the $\eta_{0}$ and $\lambda$ increased remarkably not only with increasing fibril content, but also with increasing fibrillar aspect ratio. This confirms that the fibrillar aspect ratio greatly affected the relaxation time of PP matrix; and higher aspect ratio was more effective to form a physical entangled network.

Fig. 8 illustrates the uniaxial extensional viscosity, $\eta_{\mathrm{E}}+(t, \dot{\varepsilon})$ at a extensional strain rate of $0.05 \mathrm{~s}^{-1}$ for the neat PP, PP containing 5 wt $\%$ PA66 with spherical domains (NB), and PP containing $5 \mathrm{wt} \%$ fibrillated PA66 with various aspect ratios (AR73, (a)
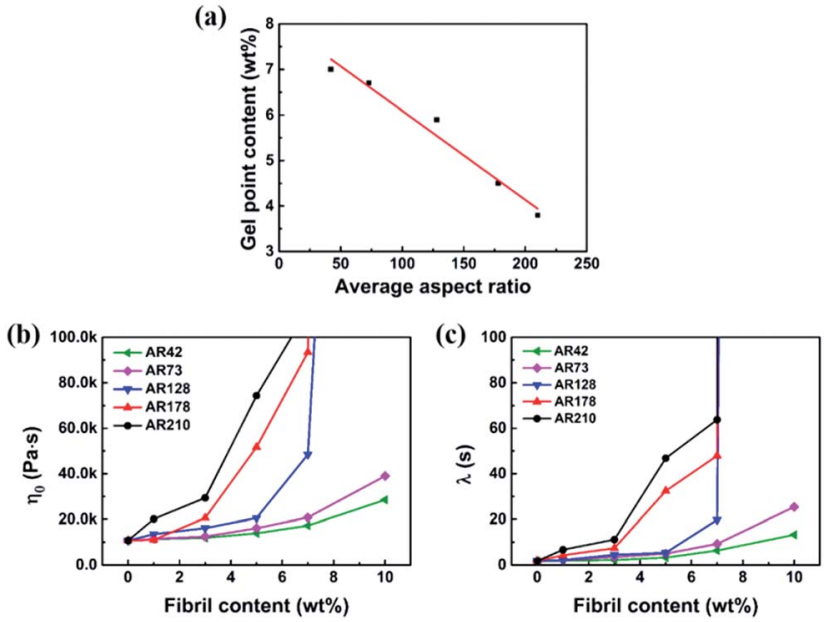

Fig. 7 (a) Linear plots of the gel point content versus fibrillar aspect ratio, (b) the zero shearing viscosity $\eta_{0}$ and (c) relaxation time $\lambda$ matched with the complex viscosity curve of five samples with different PA66 aspect ratio.

AR128 and AR210). The solid line in the figure is to show the linear viscoelastic prediction of extensional viscosity, $\eta_{\mathrm{E}}^{+}=$ $3 \eta^{+}(t)$. The $\eta^{+}(t)$ was the sample's shear viscosity in the linear viscoelastic region and was obtained from dynamic shear experiments at a strain rate of $0.001 \mathrm{~s}^{-1}$. The deviation from the extensional viscosity prediction was used to evaluate the degree of strain hardening. As seen in the graph, there is no strain hardening observed for the neat PP and NB samples. In contrast, the PP/PA66 MFCs exhibited pronounced strainhardening behaviors and higher-aspect-ratio fibrils induced strain-hardening much earlier. The rheopectic response of PP with PA66 fibrils suggested that the entangled network of fibrils was not readily to disentangle in response to the strain hardening. The similar phenomena were also observed by others. ${ }^{16,39}$

\subsection{Thermal properties}

The melting point and crystallization behaviour of the neat PP and PP with both the spherical and fibrillar PA66 domains are studied and shown in Fig. 9. It can be seen from Fig. 9a that the inclusion of PA66 increases the melting point of PP, especially for the PP with fibrillar PA domains. For instance, the melting points of PP were enhanced from $162.33^{\circ} \mathrm{C}$ to $164.27^{\circ} \mathrm{C}$ and to $169.31{ }^{\circ} \mathrm{C}$ when spherical (NB) and fibrillar PA66 domains (AR210) were added, respectively. Furthermore, the melting temperatures of PP phase in AR73, AR128 were 165.74 and 166.16, respectively. Obviously, there was a moderate increase in the melting temperature of PP phase in all microfibrillar composites compared to neat PP and NB. The formation of shish-kebab crystalline structure and transcrystalline layer of PP around PA66 may contribute to the improved melting temperature of PP phase. ${ }^{40}$

The PA66 domain morphology had a significant effect on PP's crystallization temperature during the cooling process (Fig. 9b). The crystallization temperature of the neat PP was around $110.90{ }^{\circ} \mathrm{C}$ and they were $117.85{ }^{\circ} \mathrm{C}, 120.67{ }^{\circ} \mathrm{C}$, 


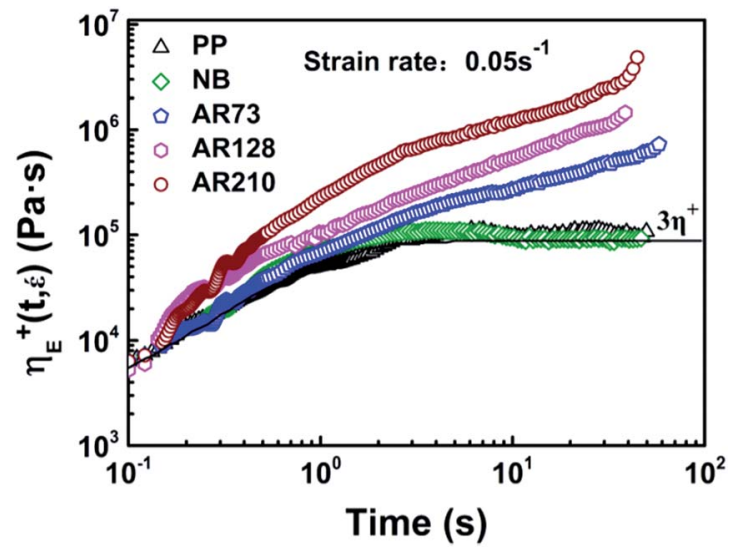

Fig. 8 Uniaxial extensional viscosity of PP, PP/spherical-PA66 (97/5 wt\%) and PP/fibrillated-PA66 blends (97/5 wt\%) with various fibrillar aspect ratio.

$121.15{ }^{\circ} \mathrm{C}$, and $121.5{ }^{\circ} \mathrm{C}$ for $\mathrm{NB}, \mathrm{AR} 73$, AR128, and AR210 samples, respectively. The presence of PA66 phase shifted the crystallization temperature of PP to higher levels considerably due to the heterogeneous crystal nucleation effect of the PA66. Compared to the unstretched PA domain, PA66 fibrils improved the crystallization kinetics of PP more significantly. This is because fibrillar domain had a larger specific surface area than spherical one, thus providing more heterogeneous crystal nucleation sites. Furthermore, the thermal properties parameters such as, melting temperature $\left(T_{\mathrm{m}}\right)$, peak or maximum crystallization rate temperatures $\left(T_{\mathrm{p}}\right)$, melting enthalpy $\left(\Delta H_{\mathrm{m}}\right)$ and percentage of crystallinity $\left(X_{\mathrm{c}}\right)$ are tabulated in Table 2 . The crystallinity of PP phase was slightly shifted to higher values with an increase in fibrillar aspect ratio. The transcrystallization effect could be the reason for an increase in $X_{\mathrm{c}}$ for PP/PA66 MFCs which possesses long PA66 microfibrils. In addition, more heterogeneous crystal nuclei could be induced by a larger specific surface area from higher aspect ratio fibrils.

\subsection{Mechanical properties}

Uniaxial tensile properties and izod impact test were conducted to understand the reinforcing potential of PA66 fibrils for PP with different content and aspect ratio (Fig. 10). The reinforcing effect in a composite system is related to many
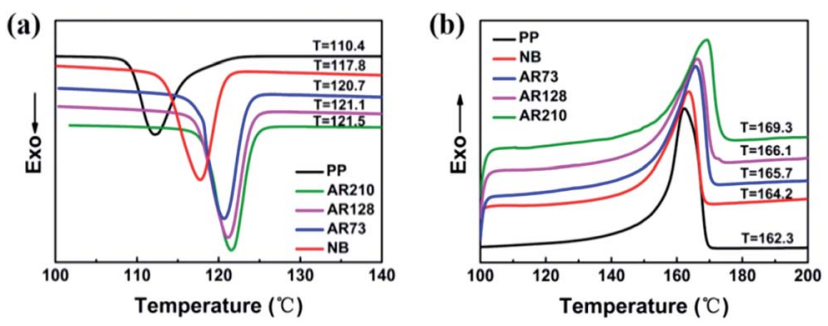

Fig. 9 (a) DSC thermograms and (b) nonisothermal crystallization curves of PP phase in the neat PP, PP/PA66 normal blend and PP/PA66 MFCs.
Table 2 The thermal properties of PP phase in neat PP, NB and PP/ PA66 MFCs with different aspect ratios

\begin{tabular}{lllll}
\hline Samples & $T_{\mathrm{m}}\left({ }^{\circ} \mathrm{C}\right)$ & $T_{\mathrm{p}}\left({ }^{\circ} \mathrm{C}\right)$ & $\Delta H_{\mathrm{m}}\left(\mathrm{J} \mathrm{g}^{-1}\right)$ & $X_{\mathrm{c}}(\%)$ \\
\hline PP & 162.3 & 110.4 & 101 & 48.3 \\
NB & 164.2 & 117.8 & 69.3 & 41.26 \\
AR73 & 165.7 & 120.6 & 85.7 & 51.2 \\
AR128 & 166.1 & 121.1 & 88.1 & 52.6 \\
AR210 & 169.3 & 121.5 & 82.4 & 56.3 \\
\hline
\end{tabular}

factors, such as amount of fiber, the aspect ratio, length distribution of the fiber, amount of entangling points of the fibers, and the adhesion between the fiber and the matrix. Fig. 10a shows the tensile and impact strength of PP/PA66 blends as a function of fibril content at a fixed fibrillar aspect ratio of 210 . The tensile and impact strength increased sharply before the PA66 content rose to $10 \mathrm{wt} \%$, after which only slight improvement was observed. This phenomenon was mainly due to the fact that the degree of dispersion and distribution of PA66 decreased and the increased agglomeration with the growth of PA66 content.

In order to study the effect of PA66 aspect ratio on the mechanical properties of PP, the PA66 content was fixed at 10 wt $\%$ and the detailed results were present in Fig. 10b and Table 3. As the PA morphology changes from spheres-ellipsoid-short microfibrils to long microfibrillar structure, the tensile strength and impact strength increased significantly. Specifically, as the fibrillar aspect ratio increased from 1 to 225 , the tensile strength and impact strength increased by $17.81 \%$ and $64.41 \%$, respectively.

We suggest that for higher PA66 fibrillar aspect ratio, the entangled network occurred more easily and stronger during compounding, which is primarily responsible for a significant improvement of tensile strength and impact strength of PP matrix. Also, with the amount of fibrils added, adhesion between the fibrils and the matrix can affect the reinforcement of a composite system. Herein, the abundance of the long PA66 fibrils is acting as excellent stress transfer neurogen, which contribute to the enhancement of the mechanical properties. Additionally, there is a strong possibility for the formation of a shish-kebab structure and transcrystalline layer of PP around PA66 in the case of samples with high aspect ratio, and this structure improved the adhesion between both phases obviously. ${ }^{41}$
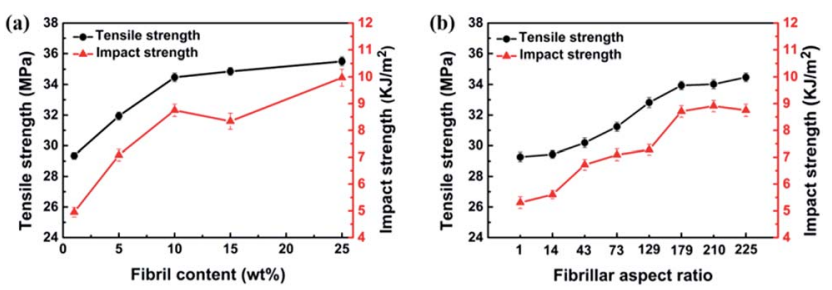

Fig. 10 The tensile and impact properties under (a) different PA66 content with aspect ratio of 210 and (b) different fibrillar aspect ratio with PA66 content of 10 wt\%. 
Table 3 The mechanical properties of NB and PP/PA66 microfibrillar composites

\begin{tabular}{lllll}
\hline Sample code & Average aspect ratio & Tensile strength $(\mathrm{MPa})$ & Tensile modulus (GPa) & Impact strength kJ m $^{-2}$ \\
\hline MC-LT & $73 \pm 10$ & $31.24 \pm 0.30$ & $1.26 \pm 0.09$ & $7.09 \pm 0.22$ \\
MC-MT & $128 \pm 8$ & $32.82 \pm 0.33$ & $1.37 \pm 0.12$ & $7.28 \pm 0.20$ \\
MC-HT & $42 \pm 23$ & $30.19 \pm 0.32$ & $1.22 \pm 0.10$ & $6.72 \pm 0.20$ \\
LC-MT & $13 \pm 5$ & $29.44 \pm 0.25$ & $1.08 \pm 0.07$ & $5.61 \pm 0.15$ \\
HC-MT & $210 \pm 11$ & $34.02 \pm 0.31$ & $1.73 \pm 0.08$ & $8.91 \pm 0.21$ \\
HC-LS & $178 \pm 13$ & $33.94 \pm 0.27$ & $1.65 \pm 0.05$ & $8.71 \pm 0.24$ \\
HC-HS & $225 \pm 20$ & $34.47 \pm 0.28$ & $1.92 \pm 0.07$ & $8.73 \pm 0.23$ \\
NB & 1 & $29.26 \pm 0.31$ & $0.91 \pm 0.11$ & $5.31 \pm 0.22$ \\
\end{tabular}

\section{Conclusions}

In this study, we show that in situ PA66 fibrils can be produced using a triangle arrayed triple-screw extruder (TTSE) at a temperature between the melting points of two phases, without extra stretching. The direct, simple, and fast extrusion process exhibited great potential in preparing microfibrillar polymer blends for industrial production. Compared to the neat PP and PP blend with spherical PA66 domain, PP/PA66 MFCs showed significantly higher storage modulus and complex viscosity at low frequency range, and greater Cole-Cole circle radius. In addition, these rheological characteristics dramatically increased with the growth of fibrillar aspect ratio. The Winter-Chambon analysis revealed that the critical gel point concentration decreased with an increase of fibrillar aspect ratio due to increased interfacial area and higher degree of entanglement. It indicated that, with higher-aspect-ratio PA66 fibrils, a three-dimensional physical entanglement network in PP can be formed at low PA66 content. The incorporation of PA66 fibrils enhanced PP's melting temperature and crystallization kinetics due to significant heterogeneous nucleation. Compared to the PA66 spherical domain, PA66 fibrillar structure had more profound effect on improving PP's tensile strength and modulus and impact strength. Furthermore, these investigated properties were increased with an increase in fibrillar aspect ratio.

\section{Acknowledgements}

The authors gratefully acknowledge the financial support of the National Key Research and Development Program of China (2016YFB0302201) and the National Natural Science Foundation of China (51273019).

\section{References}

1 W. Y. Hsu, T. D. Gierke and C. J. Molnar, Macromolecules, 1983, 16, 1945.

2 D. Wang and G. Sun, Eur. Polym. J., 2007, 43, 3587.

3 K. Dai, Z. M. Li and X. B. Xu, Polymer, 2008, 49, 1037.

4 L. S. Wang, H. C. Chen, Z. C. Xiong, X. B. Pang and C. D. Xiong, Macromol. Mater. Eng., 2010, 295, 381.

5 I. Kelnar, I. Fortelný, L. Kaprálková, J. Kratochvíl, B. Angelov and M. Nevoralová, J. Appl. Polym. Sci., 2016, 133, 43061.
6 J. Dong, Y. Qi, J. Sun, L. Wei and S. Qin, J. Appl. Polym. Sci., 2016, 133, 43797.

7 X. C. Xia, Q. P. Zhang, L. Wang, J. M. Feng and M. B. Yang, Macromol. Chem. Phys., 2014, 215, 1146.

8 J. Wang, X. Zhang, T. Zhao, L. Shen, H. Wu and S. Guo, J. Appl. Polym. Sci., 2014, 131, 40108.

9 S. R. Raghavan and J. F. Douglas, Soft Matter, 2012, 8, 8539. 10 Z. Zhao, Q. Yang, Z. Xiang, M. Kong, D. Tang, Y. Huang, X. Liao and Y. Niu, Polym. Adv. Technol., 2015, 26, 1275.

11 A. R. Kakroodi, Y. Kazemi, W. D. Ding, A. Ameli and C. B. Park, Biomacromolecules, 2015, 16, 3925.

12 A. Rizvi, C. B. Park and B. D. Favis, Polymer, 2015, 68, 83.

13 L. Yang, J. Su, Q. Yang, T. Zhang, Z. Zhao, Y. Huang and X. Liao, J. Mater. Sci., 2016, 51, 10386.

14 N. Dencheva, Z. Denchev, N. Stribeck, M. Motovilin, A. Zeinolebadi, S. S. Funari and S. Botta, Macromol. Mater. Eng., 2013, 298, 1100.

15 T. Yokohara, S. Nobukawa and M. Yamaguchi, J. Rheol., 2011, 55, 1205.

16 A. Rizvi and C. B. Park, Polymer, 2014, 55, 4199.

17 A. Rizvi, A. Tabatabaei, M. R. Barzegari, S. H. Mahmood and C. B. Park, Polymer, 2013, 54, 4645.

18 W. Liu, M. Nie and Q. Wang, RSC Adv., 2014, 4, 47793.

19 Z. Zhao, Q. Yang, M. Kong, D. Tang, Q. Chen, Y. Liu, F. Lou, Y. Huang and X. Liao, RSC Adv., 2015, 5, 43571.

20 K. Jayanarayanan, T. Jose, S. Thomas and K. Joseph, Eur. Polym. J., 2009, 45, 1738.

21 F. Chambon and H. H. Winter, J. Rheol., 1987, 31, 683.

$22 \mathrm{H}$. H. Winter, P. Morganelli and F. Chambon, Macromolecules, 1988, 21, 532.

23 V. Mittal, Functional polymer blends: synthesis, properties, and performance, ed. V. Mittal, Taylor \& Francis Group, New York, 2012, p. 233.

24 S. B. Kharchenko, J. F. Douglas, J. Obrzut, E. A. Grulke and K. B. Migler, Nat. Mater., 2004, 3, 564.

25 S. Fakirov, D. Bhattacharyya and R. J. Shields, Colloids Surf., A, 2008, 313, 2.

26 K. Jurczuk, A. Galeski and E. Piorkowska, Polymer, 2013, 54, 4617.

27 X. Z. Zhu, H. Q. Yuan and W. Q. Wang, J. Mater. Process. Technol., 2009, 209, 3289.

28 X. Z. Zhu, Y. J. Xie and H. Q. Yuan, Polym.-Plast. Technol. Eng., 2007, 46, 401. 
29 K. Yang, C. Xin, D. Yu, B. Yan, J. Pang and Y. He, Polym. Eng. Sci., 2015, 55, 156.

30 Z. M. Li, M. B. Yang, B. H. Xie, J. M. Feng and R. Huang, Polym. Eng. Sci., 2003, 43, 615.

31 J. A. Covas, O. S. Carneiro and J. M. Maia, Int. J. Polym. Mater., 2001, 50, 445.

32 W. D. Ding, T. Kuboki, A. Wong, C. B. Park and M. Sain, $R S C$ Adv., 2015, 5, 91544.

33 D. F. Hodgson and E. J. Amis, Macromolecules, 1990, 23, 2512.

34 R. Muller, E. Gerard, P. Dugand, P. Rempp and Y. Gnanou, Macromolecules, 1991, 24, 1321.
35 J. Stange, C. Uhl and H. Münstedt, J. Rheol., 2005, 49, 1059. 36 J. Tian, W. Yu and C. Zhou, Polymer, 2006, 47, 7962.

37 J. Koszkul and J. Nabialek, J. Mater. Process. Technol., 2004, 157-158, 183.

38 N. P. T. O'Connor and R. C. Ball, Macromolecules, 1992, 25, 5677.

39 M. Yamaguchi, K. Fukuda, T. Yokohara, M. A. B. M. Ali and S. Nobukawa, Macromol. Mater. Eng., 2012, 297, 654.

40 H. Quan, Z. M. Li, M. B. Yang and R. Huang, Compos. Sci. Technol., 2005, 65, 999.

41 J. K. Kim and Y. W. Mai, Compos. Sci. Technol., 1991, 41, 333. 